\title{
Assessing Online-form Complexity for the Development of Assistive Technologies for Older Adults
}

\author{
Arthur G. Money, Senaka Fernando, Lorna Lines and Anthony D. Elliman \\ Department of Information Systems and Computing, Brunel University, Uxbridge, UK
}

\begin{abstract}
Although cognitive declines occur as a natural product of the ageing process, the majority of online-forms do not cater specifically for the needs of older adult users. As a consequence, online-forms pose significant usability challenges to this target user group. The Delivering Inclusive Access to Disabled and Elderly Members of the community (DIADEM) project aims to develop a plug-in to a web browser that adapts existing online-form content so that it is more accessible and usable for older adults with cognitive decline. In order to identify requirements for developing the DIADEM application, it is necessary to observe users interacting with onlineforms, and identify the usability challenges that occur as a result of this. However, the format and functionality of online-form content presented on the web varies greatly. Identifying a representative sample of online-forms that may be presented to users within a trials setting to elicit key usability challenges, has proved to be a non-trivial task. Consequently, we have developed a set of Bespoke Online-form Selection (BOFS) criteria which are used to help identify appropriate and representative candidate online-forms that may be used within the user trials setting to formulate initial requirements for developing the DIADEM application. In the context of the DIADEM project, BOFS has proved to be a valuable tool which has been used to successfully identify online-forms for use in our user trials. This paper presents the BOFS criteria, and shows how these are aligned with cognitive declines that are typically presented by the older adult user group, and demonstrates how BOFS has been of value within the context of the DIADEM project.
\end{abstract}

Keywords: older adults, cognitive decline, online-form complexity, assistive technology, complexity

\section{Introduction}

In recent years, older adults have become one of the fastest growing groups of web-service users [Fernando et al. 2009, Money et al. 2011]. It is widely accepted that cognitive decline occurs as a natural product of the ageing process [Peterson et al. 1997], and is unavoidable in old age [Christensen 2001]. With an ageing European population [Kleinberger et al. 2007], it is not surprising that the number of individuals in Europe presenting with cognitive decline is on the increase [Disability-Rights-Commission 2005]. A large proportion of these users, particularly those living independently, are likely to require assistance from both public and private agencies to support independent living tasks such as grocery shopping, accessing social services, paying utility bills, and managing and accessing healthcare [Holzinger et al. 2008]. Government initiatives within the European Union (EU) have led to the wide-spread adoption of web-services as a means to improve the accessibility and efficiency of government welfare services for their citizens. However, older adults are known to have less experience in the use of web-services compared with younger adults [Morrel and Mayhorn 2000], and coupled with increased levels of cognitive decline, older adults are at a distinct disadvantage when attempting to effectively access such services [Fuchsberger 2008, Lines et al. 2006].

There is a growing need to develop assistive technologies that enable older adults to access web-services more effectively and efficiently. There is a wide variety of services that such people might use including, online shopping, purchasing of travel tickets, applying for parttime work to boost weekly income, requesting welfare support, and booking hospital appointments. In every case, the user needs to go through several web-pages "filling in an onlineform" to get the desired result. It is important 
to distinguish, what we as developers, perceive to be an online-form and what users perceive. Technically, each new HTML page is a separate form. However, a user accustomed to turning the pages of paper-based forms, is likely to perceive such a sequence as a single, multipaged form. In this paper we are concerned with user perceptions, and therefore references to an "online-form" should be read as any sequence of pages that the user will perceive as soliciting the data associated with a single transaction.

The problem then is to find a basis for reviewing the wide range of possible online-form filling transactions that are presented on the web, to identify a representative sample of content that may be utilised, within a user trials setting, to formulate design guidelines for the older adult target user group. In any bounded piece of usability research, it is necessary to consider whether the forms used in the actual user trials are typical of other online activities, particularly if the results are to be generalisable. As explained in the next section, the existing research has tended to use methods that are dependent on the responses of user panels and therefore are not transferable to desktop evaluation across a wide range of sites. Furthermore, such methods are often time consuming and costly, requiring a preliminary series of user trials to identify a representative sample of online-forms, and then a subsequent main series of user trials to identify design guidelines for online-form content.

The aim of this paper is to present the Bespoke Online-form Selection (BOFS) criteria, which serve as an aid in the process of identifying appropriate online-forms for use within the main series of user trials. The BOFS criteria provide an alternative means of measuring the relative complexity of online-form content, as may be perceived by cognitively impaired older adult users, without requiring an additional round of preliminary user trials. The remainder of this paper is structured as follows. In Section 2, the DIADEM project is described and existing web-content usability evaluation methods are reviewed to establish the need for a new online-form complexity metric. In Section 3 the research methodology used in this study is presented. Section 4 presents the BOFS criteria. Section 5 demonstrates how the BOFS criteria may be applied to a range of online-form content in order to gain insight into their relative complexity. Section 6 reports on the effectiveness of BOFS within the context of the DIADEM project. Section 7 concludes this paper.

\section{Background}

The DIADEM project, funded by the European Commission (EC), has the primary goal of improving older adults' access to online-forms via the development of DIADEM, a plug-in to a web-browser that monitors user browsing and input activity, and adapts and personalises the online-form interface via assistive behaviour to meet the individual user's needs. A series of user trials have been carried out in the UK, Italy and Norway, so that a comprehensive list of functional and usability requirements may be identified for the development of the DIADEM application. The aim of the trials was to observe older adults as they interact with online-forms, and identify the usability challenges that arise as a result of these interactions. The results would then be used to formulate requirements for the DIADEM application. However, in order to carry out the user trials, it was necessary to identify a discrete set of candidate onlineforms that were likely to elicit a broad range of key usability challenges for the cognitively impaired older adult user group. Therefore, it was important that the content and functionality of the candidate online-forms used within the trials was sufficiently complex, to ensure that a wide range of usability challenges were identified as a result of users interacting with them. Identifying such online-forms has proved to be somewhat of a challenge, since there appears to be a gap in the existing literature in providing guidance for carrying out such a task. Consequently, we have developed a set of Bespoke Online-forms Selection (BOFS) criteria, which provide a means of assessing the complexity of online-forms, according to five cognitive domains that are known to decline as a result of ageing, as indicated by the Addenbrooke's cognitive examination [Mioshi et al. 2006]. It is assumed that the more complex the online-form is in terms of content and functionality, the more likely it is to elicit a broad range of key usability challenges. Therefore, by applying BOFS to a range of online-forms and comparing the results, the relative complexity of online-forms may be assessed, which in turn may be used to identify a short list of comparatively complex candidate online-forms that may be used within 
the user trials setting. BOFS has been used successfully within the DIADEM project, and has provided a valuable means of identifying those online-forms which appear to be most complex, and thus most appropriate for use within our research.

\subsection{Existing Research}

Currently the most common approach to measuring complexity of web-content is carried out in the usability evaluation research domain [Holzinger 2005]. Usability evaluation methods consider three user-based criteria; the effectiveness, the efficiency, and the satisfaction [de Kock et al. 2009]. Several studies have been carried out to assess these usability criteria. For example, efficiency has been evaluated by measuring the number of successful "hits" identified in a testing session [Hvannberg et al. 2007], and the time it takes for a user to navigate from one hypertext node to the next [Cribbin and Chen 2001], by measuring the user's heart rate variability whilst carrying out specific computer-based tasks [Iszo and Lang 2000], and by counting the number of mouse-clicks while carrying out browsing tasks [Drucker et al. 2002]. Bayles [2002] evaluated effectiveness by measuring the levels that users recalled information from web-based banner advertisements, and Dumais et al. [2002], by measuring the number of web-based tasks the user failed to complete. McGrenere et al. [2002] evaluated satisfaction by asking users to report their perceived annoyance level, and Chevalier and Bonnardel [2007] measured the number and nature of constraints that are verbalised by users whilst interacting with web-content. These are just a few of a large number of studies that have been carried out within the usability evaluation domain, see Hornbaek [2006] for a more comprehensive review of 180 contemporary research articles in this area.

Web-content may also be evaluated via heuristic inspection methods [Ling and Salvendy 2009, Te'eni et al. 2007]. Some of these methods include heuristic evaluation, cognitive walkthroughs, formal usability inspections, and the pluralistic usability walkthrough [Hollingsed and Novick 2007]. With the exception of heuristic evaluation, all of these methods require the user to undertake interaction tasks in order to evaluate the usability of the interface $[\mathrm{Heo}$ et al. 2009]. Heuristic evaluation, on the other hand, typically involves a small number of usability experts who refer to a set of generic web-content usability principles, and inspect the user interface to estimate the significance of usability issues accordingly [Ling and Salvendy 2009]. Popular interface design principles used for such an exercise include the Multiple Heuristics Evaluation Table (MEHT), which combines a number heuristic evaluation methods into one evaluation table [Atkinson et al. 2007]. Frokjaer and Hornbaek's [2008] Metaphors of Human Thinking (MOT) method, which uses metaphors grounded in psychological theory to drive heuristic evaluation forward. Buxton et al. [2009] present the Integrated Stakeholder Usability Evaluation Process (ISUEP) for evaluating mission critical decision support systems. ISUEP makes it possible, via a formal process of filtering and prioritisation, for large teams (16 or more people) to carry out effective heuristic evaluations. Faaborg and Schwartz [2010] propose the development of a distributed heuristic evaluation function that may be integrated into standard Web browsing interfaces such as Mozilla. Seffah et al. [2006] present the Quality in Use Integrated Measurement (QUIM) usability assessment model, which consolidates a wide range of usability assessment methods and standards into a single model of usability measurement. This can be used by experts and novices to carry out detailed evaluations of software usability. Some pioneering work in this field includes Schneiderman's [1998] eight "golden rules" of interface design, and Nielsen's [1994] 10 usability heuristics. Some examples of where these methods have been used include a study presented by Naseem [2005], who used four usability experts and Nielsen's [1994] 10 heuristics to evaluate and redesign an open learning website. Tang et al. [2006], also used a combination of these heuristics to carry out an evaluation involving three usability experts, to inspect a prototype telemedicine system. It was found that the improvements to the user interface made as a consequence of the heuristic evaluation resulted in more effective and efficient interactions with the paramedics that used the web-based system.

During the web-content development phase, design guidelines are also used to ensure the content is usable by the target user group. The World Wide Web Consortium Web Content Accessibility Guidelines (WCAG) [W3C 2008], 
are made up of 12 guidelines organised under four principles which can be used by web-page authors and designers to ensure web-content is developed to be accessible for people with disabilities in general. The United States general services administration section 508 [1998] also provides guidelines that should be adhered to for supporting inclusive access for disabled users.

In terms of design guidelines specifically for older adult users, Hawthorn [2000] noted that there was lack of research that focused on design of interfaces that specifically cater for the needs of this target user group. Since then, a number of web-content accessibility guidelines for older adults have been presented in the research literature. Based on an extensive review of research concerning age-related changes in vision, Echt [2001] proposed 19 guidelines for the effective presentation of web-based health information for older adult users. Each of these guidelines were presented in one of three categories: typography, layout, and navigation. Although these guidelines were presented in the context of delivering web-based health information, much of this work appeared to be relevant to web-content more generally. Consequently many of the guidelines appear to have been incorporated into the 25 guidelines developed by The National Institute of Ageing (NIA) for web-content targeting users aged $60+[$ Morrell et al. 2002]. These guidelines have been derived from a variety of previous research, in the human-factors, cognition, ageing and print materials research domains. The guidelines are recognized as being a comprehensive and empirically-based set of guidelines, which, when applied to web-content, are shown to be effective in improving the "senior-friendly" nature of web-content [Morrell 2005]. Kurniawan and Zaphir [2005] produced guidelines based on previous literature, which were refined and categorized through a series of focus groups and card sorting exercises. Nielsen \& Norman [2002] also produced guidelines for older adults, but rather than sourcing these from literature, the guidelines were derived from a series of trials carried out with older adults aged $65+$. The result was 46 design guidelines.

Paper-based form filling has been a part of everyday life for decades, however web-based forms (online-forms) are becoming increasingly common, and an essential part of e-government and e-commerce online web services. Unlike generic web-content, in general, online-forms present specific usability challenges to the user, not least due to their predominantly question and answer based nature [Arch 2008, Lines et al. 2007]. Although there appears to be some research literature presenting accessibility design guidelines for older adults relating to webcontent in general, there seems to be very little research presenting guidelines for older adults relating specifically to online-forms [Sayago and Blat 2007]. Lines et al. carried out two small scale studies, in the UK [Lines, Ikechi and Hone 2007, Lines 2004]. The most recent of these Lines et al. [2007], validating the results from a previous study [Lines 2004], carried out trials using financial, housing, and welfare online-forms. As a result, 13 e-Government online-form design guidelines were presented. Sayago and Blat [2007] carried out a small scale study with seven Spanish users. The study focused on specific hypotheses, and used a short generic online-form. Although the output of this study did not result in a comprehensive set of design guidelines, the findings revealed that older adult users prefer checkboxes and radio-buttons as opposed to list-boxes, and that users preferred required and optional fields to be grouped into two separate sections.

Despite much valuable research, there seems to be a lack of literature that supports the immediate needs of the DIADEM project: To identify, in a timely fashion, an appropriate sample of online-forms for use within user trials setting, to identify the key usability challenges faced by the older-adult user group [Holzinger et al. 2008]. Perhaps the reason for this is that the overwhelming majority of usability research is trial-based, and takes place with the user present. Therefore, existing research does not tend to prescribe tools for the comparatively rapid, pragmatic, but consistent assessment of online-forms that allow assessment to be carried out directly on the online-form, as opposed to directly via the user's experience of interacting with online-form content. Furthermore, existing research tends not to be specific to onlineform content and the older adults, and therefore does not provide a suitable basis on which to evaluate the complexity of online-form content for the target user group in question. In direct response to the needs of our project, BOFS has been developed. 


\section{Research Methodology}

The aim of developing BOFS was to serve primarily as an aid to identifying appropriately complex online-forms which could subsequently be used in the forthcoming trials process. It was therefore necessary to develop BOFS within the time-constraints of the project, whilst maintaining methodological rigour in terms of its development. In order to achieve this, BOFS was developed via a systematic consultation process, involving experts in online-form design and age-related cognitive impairments. This involved experts reviewing existing research in these research domains, and through a process of discussion and consensus building, developing a set of online-form criteria that may be used to estimate the relative complexity of online-forms for users with age-related cognitive impairments. Often a significant challenge is sourcing experts to take part in such an exercise [Papazafeiropoulou et al. 2002]. However, a key advantage of DIADEM being a panEuropean project, with multiple large-scale organisations as consortium members, was that the process of sourcing experts to contribute to the development of BOFS was a comparatively straightforward exercise. This was achieved by engaging all of the DIADEM project consortium members, and a range of their respective associated partner networks.

A total of 12 experts were involved in the BOFS development process. Brunel University provided two experts in the field of HCI and online- form design to take part in the BOFS development process. Brunel also collaborated with a local authority (Sheffield City Council) who had links to local healthcare and voluntary organisations, which made it possible to include two domain experts from the UK based University of the Third Age (U3A), and two experts from the UK based Partnership for Older People Projects (POPPS) in the BOFS development process. With a vast amount of experience in developing access for all in the eInclusion arena, two senior e-Inclusion consultants from Sheffield City Council (SCC) were also actively involved in every aspect of the BOFS development process. CSI Piemonte (an IT consortium based in Turin, Italy) provided two valuable contacts within the Piemonte regions' healthcare sector, including links with local hospitals, who provided expert clinical input. Two Norwegian IT solutions companies from Norway, both with experience of webbased e-Government applications development, provided one expert each, to advise on the technical relevance of the criteria included in BOFS. The BOFS development process is outlined in Figure 1.

As recommended in consensus building methods best practice guidelines [Black et al. 1999], prior to holding focus group sessions to develop the BOFS criteria, the experts initially shared and reviewed relating to theoretical aspects of age-related cognitive impairments. The aim was to identify an overarching categorisation of the cognitive functions that decline as

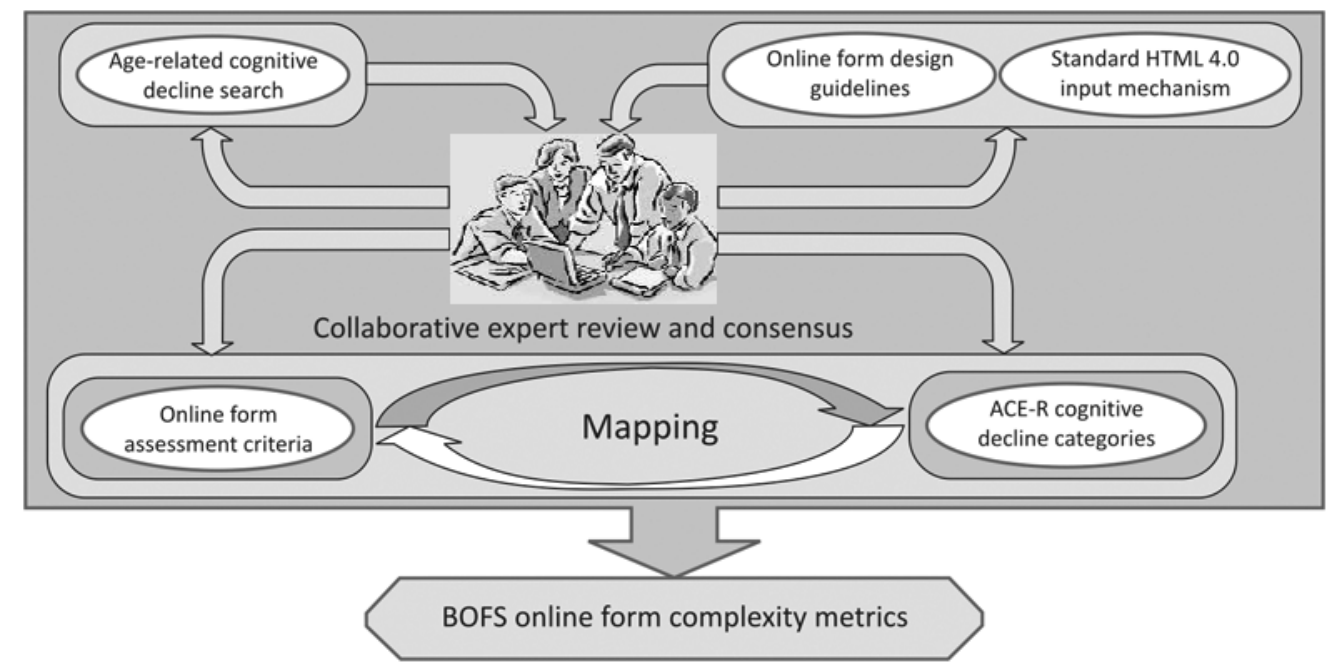

Figure 1. BOFS development process. 
a result of ageing, so that online-form functionality could be considered, categorised, and prioritised against these categories.

A range of age-related cognitive decline research, in the form of models of cognition and cognitive ability tests, were considered by the expert panel. These included the work of Albert et al [Albert et al. 1995] on the predictors of ageing, the Wechsler's Adult Intelligence Scale Revised (WAIS-R) [Welchsler 1982], the Global Deterioration Scale (GDS) for assessment of primary degenerative dementia [Reisberg et al. 1982], the Mini Mental State Examination (MMSE) [Folstein et al. 1975], and the Addenbrooke's Cognitive Examination Revised (ACE-R) [Mioshi, Dawson and Mitchell 2006]. As a result, the five cognitive impairment categories (sub-scales) used by the ACE$\mathrm{R}$ were identified as being the most appropriate and comprehensive for the purposes of the task. The ACE- $\mathrm{R}$ is a comparatively new, brief, sensitive, and inexpensive screening tool [Mioshi, Dawson and Mitchell 2006], which fully incorporates the cognitive tests such as the MMSE. It is also considered to be more sensitive than the MMSE alone, and has been shown to be more effective in identifying early cognitive dysfunction/mild cognitive impairments [Larner 2006].

In order to identify and categorise online-form functionality that may cause challenges to cognitively impaired older adults, a range of onlineform design guidelines were considered: these included The National Institute of Ageing (NIA) web-content design guidelines developed for users aged 60+ [Morrell, Dailey, Stoltz-Loike, Feldman, Mayhorn, Echt and Podany 2002], which are recognised as being a comprehensive and empirically-based set of guidelines, which are shown to be effective in improving the "senior-friendly" nature of web-content [Morrell 2005]; Nielsen and Norman's 46 guidelines [Nielsen and Norman 2002] also relating to development of web-content for older adults, but rather than sourcing these from literature, the guidelines were derived from a series of trials carried out with older adults aged 65+; Guidelines developed by Lines et al. [Lines, Ikechi and Hone 2007, Lines 2004] which are specifically for the development of online-forms for older adults were also considered. The HTML input mechanisms, as identified by Miller and Jarrett [Miller and Jarrett 2001] were also considered in the process. Furthermore, a range of example online-forms were used as a point of reference throughout, to provide examples of online-form functionality to aid the decision making process. By systematically working through the online-form design guidelines, and interacting with a range of online-form content, a short list of online-form assessment criteria relevant to the project needs was developed, and mapped to the ACE-R cognitive impairment categories, in order to substantiate the criteria's relevance to the cognitively impaired older adult user group. In the next section, the BOFS criteria are presented.

\section{The BOFS Criteria}

The Bespoke Online-form Selection (BOFS) criteria were developed to assist in the task of identifying online-forms that would be considered complex for the older-adults to complete. BOFS produces a range of numeric values that may be used to estimate the relative "level of complexity" of a given sample of online-forms. Due to the small amount of existing research that evaluates online-form complexity for this user group, it seems that formal methods for analysing online-form complexity have yet to be addressed. Consequently, the BOFS criteria were developed that provide a structured and straightforward means of evaluating the complexity of online-forms for cognitively impaired older adults. As described in the previous section, all criteria were derived by an expert panel, in light of the five sub-scales (which represent the five known cognitive domains that are known to be affected as a result of cognitive decline) used by the Addenbrooke's cognitive examination (ACE-R) [Mioshi, Dawson and Mitchell 2006]. Therefore, each of the BOFS criteria were considered to present challenges relating to one or more of the ACE-R cognitive impairment categories, and therefore included as assessment criteria within BOFS.

The ACE- $\mathrm{R}$ is a comprehensive cognitive ability screening tool, which is now becoming widely used to identify users that may be showing signs of cognitive decline. The cognitive domains that decline in this user group, as indicated by the ACE-R examination, are as follows:

- ACE-RI - Attention and orientation: Users have impaired skills related to orientation 
within time and space, and maintaining attention for extended periods of time.

- ACE-R2 - Memory: Impaired memory recall, in the short term, and short to medium term.

- ACE-R3-Fluency: Impaired fluency of categorisation (for example categorising words), impaired inference, assimilation, interpretation, and elaboration of related concepts.

- ACE-R4 - Language: Impaired ability to recall low frequency words and irregular words.

- ACE-R5 - Visuospatial: Impaired visual intuition, and interpreting or implying meaning from visual cues.

The following is a description of the criteria incorporated within BOFS. Each of the criteria is associated with one or more of the ACE-R cognitive decline domains listed above. Table 1 provides a summary of the BOFS criteria and how each of these correspond with the ACE-R cognitive impairment categories.

BOFS aims to provide a generic solution, which assesses a range of generic online-form criteria. As can been seen in Table 1, some of these criteria include: the number of pages in the online-form; the amount of scrolling required within a form; whether the online-form gives an idea of the users location within the context of the whole form, and so forth. In addition to global navigation elements, Miller and Jarrett [2001] identify five HTML 4.0 input mechanisms, which are also taken into account by BOFS. These are:

\section{- Drop-down boxes (BOFS8a)}

- Radio buttons (BOFS8b)

- Check boxes (BOFS8c)

- Hyperlinks (BOFS8d)

- Type-in boxes (BOFS8e)

Since online-forms are primarily concerned with users inputting data, BOFS also allows onlineform complexity to be assessed according to these five types of input mechanism, and thus can be applied to any online-form that has been designed in standard HTML 4.0 format, regardless of the genre of the online-form or indeed the specific subject content of the online-form. In the next section, the BOFS criteria are presented in more detail, along with how they are associated with the five ACE-R cognitive impairment categories.

\begin{tabular}{|l|l|l|}
\hline $\begin{array}{c}\text { BOFS } \\
\text { criteria }\end{array}$ & Description & ACE-R cognitive impairment category \\
\hline \hline BOFS1 & Number of pages in the form & ACE-R1, ACE-R5 \\
\hline BOFS2 & Number of pages requiring scrolling & ACE-R1, ACE-R5 \\
\hline BOFS3 & Current location (page) indicator & ACE-R1, ACE-R2, ACE-R3 \\
\hline BOFS4 & Overall location (form) indicator & ACE-R1, ACE-R2 \\
\hline BOFS5 & Number of questions in the form & $\begin{array}{l}\text { ACE-R1, ACE-R2, ACE-R3, ACE-R4, } \\
\text { ACE-R5 }\end{array}$ \\
\hline BOFS6 & Maximum number of possible responses & ACE-R2, ACE-R3 \\
\hline BOFS7 & Number of responses requiring additional information & ACE-R1, ACE-R2 \\
\hline BOFS8a & $\begin{array}{l}\text { - Number of drop-down boxes in the form } \\
\text { - Number of drop-down boxes requiring scrolling } \\
\text { - Number of options in drop-down boxes }\end{array}$ & ACE-R2, ACE-R3 \\
\hline BOFS8b & $\begin{array}{l}\text { - Number of radio-buttons in the form } \\
\text { - Number of options for radio-buttons }\end{array}$ & ACE-R2, ACE-R3 \\
\hline BOFS8c & $\begin{array}{l}\text { - Number of check-boxes in the form } \\
\text { - Number of options for check-boxes }\end{array}$ & ACE-R2, ACE-R3 \\
\hline BOFS8d & $\begin{array}{l}\text { - Number of hyperlinks/buttons in the form } \\
\text { - Number of options for hyperlinks }\end{array}$ & ACE-R1, ACE-R2, ACE-R3 \\
\hline BOFS8e & $\begin{array}{l}\text { - Number of type-in boxes in the form } \\
- \text { Number of type-in boxes requiring more than one } \\
\text { sentence }\end{array}$ & ACE-R1, ACE-R2 \\
\hline
\end{tabular}

Table 1. BOFS criteria and corresponding ACE-R cognitive impairment categories. 


\subsection{BOFS and the ACE-R Cognitive Impairment Categories}

\section{BOFS1. Total number of pages in the online- form}

A high number of pages included within an online-form are likely to increase cognitive load, increase fatigue, and require the user to maintain attention for prolonged periods of time (ACER1). Any visual cues used, for example, to demonstrate to the user the form structure such as a site map, will be more complex as the number of pages increase in the form increase, to reflect the locations of the various pages, which in turn could put increased strain on users interpreting these cues (ACE-R5).

\section{BOFS2. Total number of pages that require scrolling to view entire page}

It is important that the end-users understand "where they are" within a page. If scrolling is required to complete the page, the end-user may become confused and disoriented (ACER1). By scrolling down, the end-users may also "miss" questions and not complete the page at first attempt, it may also put strains on their ability to visually understand the significance and meaning of visual cues within the form (ACE$\mathrm{R} 5)$.

\section{BOFS3. Current location (page) indicator}

When completing online-forms, it is important for the end-users to "know where they currently are" within the online-form to reduce confusion, disorientation (ACE-R1), and to support question completion. This is also important to minimise the cognitive load placed on the users, in terms of having to recall from memory what steps they took to navigate to this location (ACE-R2), and infer, based on the general content and concept structure of the form what steps they need to take to return to their original location (ACE-R3). Examples of current location identifiers are presented in Figure 2.

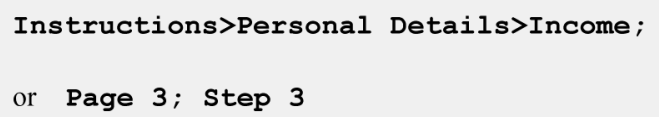

Figure 2. Example location identifiers.

\section{BOFS4. Overall location (form) indicator}

When completing online-forms, it is important for the users to know their overall location within the form, to reduce confusion, disorientation (ACE-R1). It also aids the users in knowing how much of the form they have already completed, hence reducing cognitive load in terms of having to recall from memory where they are within the context of the online-form (ACE-R2), how much they have completed, and how much they think they still have to complete. Examples of overall location identifiers are presented in Figure 3.

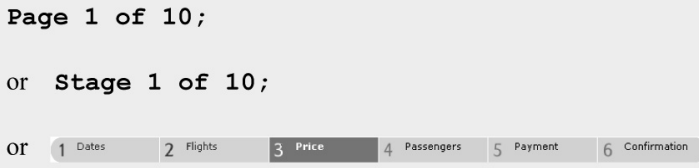

Figure 3. Overall location identifiers.

\section{BOFS5. Number of questions (include sub- questions) in form}

A high number of questions are likely to lead to greater cognitive demands being placed on the end-user, and may induce end-user frustration and fatigue (ACE-R1). The amount of text included in the online-form is likely to increase as the number of questions increases, and thus may pose more of a challenge to the user in terms of required memory recall functions in order to answer questions (ACE-R2). It is also likely to require the user to put more effort into assimilation of information, interpreting the meaning of the questions (ACE-R3), and recalling low frequency and irregular words as they occur within the online-form content (ACE-R4). The number of visual cues used within the online-form is also likely to increase as the number of questions increase, and therefore could put increased demand on visual intuition from the user ACE$\mathrm{R} 5)$.

\section{BOFS6. Maximum number of possible re- sponses}

Questions posed within a form may have a number of possible pre-determined responses (typically generated based on form processing requirements). The larger the number of possible responses to questions presented within 
a form, again the greater the likelihood of inducing greater cognitive demands in terms of memory (ACE-R2), and assimilation and elaboration of the conceptual structure of the possible answers to those questions (ACE-R3). In "simple" forms, there will typically be one response per question.

\section{BOFS7. Number of responses requiring addi- tional information}

A form is likely to be perceived as more time consuming to complete when information from other sources (such as a paper document, electronic file, a driving license etc.) is required to be able to complete the form, thus putting strain on the user's attention span (ACE-R1), as well as on their memory, should the user first need to recall where they have stored the required documents (ACE-R2).

\section{BOFS8. Input mechanisms}

According to [Miller and Jarrett 2001], HTMLbased online-forms have the functionality of enabling end-users to input answers to questions in five forms: drop-down boxes, radio buttons, check boxes, hyperlinks, and type-in boxes. Based on these five forms of input, BOFS derives the criteria presented in Figure 4 that can be additionally used to evaluate the complexity of online-forms:

\section{BOFS8a. Drop-down boxes}

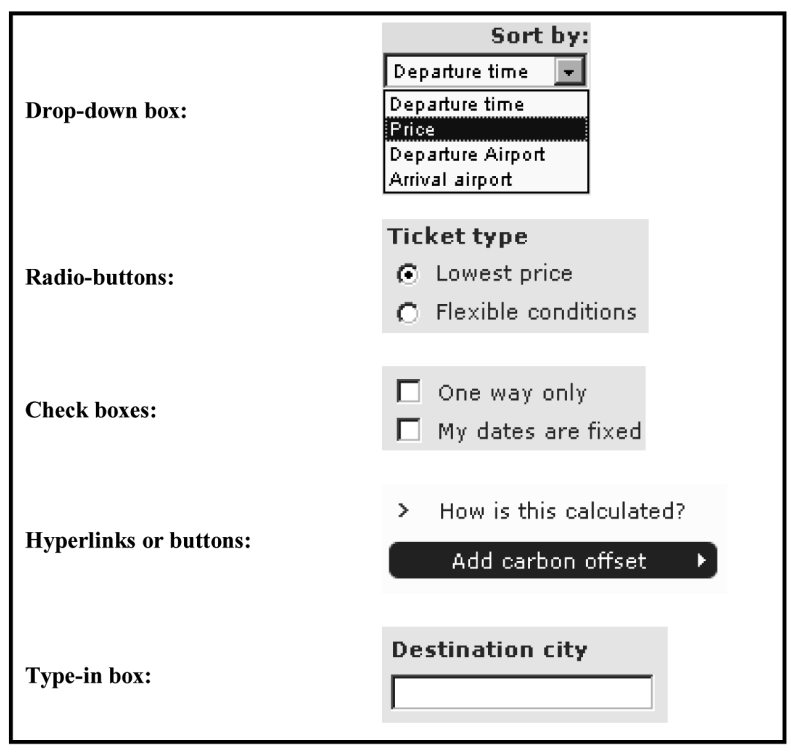

Figure 4. Examples of the five input elements sourced from British Airways travel [2008].
In analysing the use of drop-down boxes within a form, the following measures were employed:

- Total number of drop down boxes in the online-form.

- Total number of drop-down boxes that require scrolling in the online-form.

- Total number of options in drop-down boxes that require scrolling.

The number of drop-down boxes presented within each form was recorded. In addition, the number of drop-down boxes that did not present all options on the screen at the same time and required scrolling were also recorded. Where scrolling is required, there is an expectation that the end-user will be able to "find", by requiring increased levels of inference and interpretation, the options they wish to select (ACER3). However, if the options are not presented at the same time, an extra demand is made on short term memory (ACE-R2) in that the enduser will have to "recall" the options and the associated interpretations/eliminations in relation to each option. Thus, scrolling through a drop-down box, when all of the options are not visible on the screen, is likely to incur greater cognitive demands.

The number of drop-down boxes containing a specific number of options was also recorded to identify forms that used not only a high number of drop-down boxes, but also a high number of options. Selecting a response from a long list of options, all of which require some cognitive effort is likely to incur greater cognitive load than from a short list of options (ACE-R3). Table 2 provides an example of how this information was documented for a form containing a total of 7 drop down boxes with 4 comprising 3 options and 3 comprising 7 options. A similar format was used for recording BOFS $8 \mathrm{~b}, \mathrm{BOFS} 8 \mathrm{c}$, and BOFS8d.

\begin{tabular}{|l||l|}
\hline Number of drop-down boxes & 7 \\
\hline $\begin{array}{l}\text { Number of options in } \\
\text { drop-down boxes }\end{array}$ & 4 X 3 \\
\hline
\end{tabular}

Table 2. Number of drop-down boxes and options.

\section{BOFS8b. Radio buttons}

In analysing the use of radio buttons within a form, the following measures were employed: 
- Number of radio buttons in form

- Number of radio buttons grouped by number of options

The total number of questions requiring radio button input was recorded. If a "type-in" box was presented to capture end-user information (e.g. other... or if the end user has to justify their selection) this type-in box was included within the total count of "type-in" boxes as documented below. The number of radio buttons containing a specific number of options was also recorded to identify forms that used not only a high number of radio buttons, but also a high number of options. Selecting a response from a long list of options is likely to incur a proportionately greater cognitive load, in terms of interpreting and understanding and differentiating between the specific meanings of each of the options (ACE-R3). In addition, increased cognitive load in terms of short memory would also occur if the user has to hold in memory and understand all possible options, before he can choose the most appropriate of those (ACE-R2).

\section{BOFS8c. Check boxes}

In analysing the use of check boxes within a form, the following measures were employed:

- Number of check boxes in a form

- Number of check boxes grouped by number of options

The total number of questions in each form requiring check box input was recorded. If a "type-in" box was presented to capture end-user information (e.g. other. . . or if the end user has to justify their selection) this type-in box was included within the total count of "type-in" boxes as documented below. The number of check boxes containing a specific number of options was also recorded to identify forms that used not only a high number of check boxes, but also a high number of options. Again, selecting a response from a long list of options, requires added cognitive effort, which is likely to incur greater cognitive load than from a short list of options, similar to the added effort required by BOFS8b (ACE-R2 and ACE-R3).

\section{BOFS8d. Hyperlinks/Buttons}

In analysing the use of hyperlinks/buttons within a form, the following measures were employed:

- Number of hyperlinks/buttons in form
- Number of hyperlinks/buttons grouped by number of options

The total number of questions/actions requiring a response via hyperlinks/buttons presented within the entire form was recorded. The number of hyperlinks/buttons containing a specific number of options was also recorded to identify forms that used not only a high number of hyperlinks/buttons, but also a high number of options. Once again, selecting a response from a long list of options, all of which require some cognitive effort, is likely to incur greater cognitive load than from a short list of options (ACE-R2 and ACE-R3). Hyperlinks also have the potential of disorientating the user as it takes them to a different section of the form (ACER1).

\section{BOFS8e. Type-in box}

In analysing the use of type-in boxes within a form, the following measures were employed:

- Number of type in boxes

- Number of type in boxes indicating that more than one sentence/statement required.

Here the total number of type-in boxes presented throughout the form was recorded. In addition, the number of type-in boxes that would typically require more than one sentence (e.g. indicated within the form by a large text box space, else a request to providing a justification) was also recorded. Users required to complete type-in box answers may be required to maintain attention for comparatively long periods of time (ACE-R1), compared with, for example, check box answers, they may also be required to recall information from memory if they are required to provide an elaborate, free-style answers (ACE-R2).

\subsection{HTML 5, Web Forms 2.0 and Other Online Technologies}

Miller and Jarrett [2001] add a critical caveat to the selection of five types of forms element or data entry behaviour; namely "it is possible to create much more sophisticated forms with the use of other technologies such as Java applets or image maps embedded in your web page". This statement was made in 2001 and the increasing use of ActiveX components downloaded to Microsoft's Internet Explorer must be added to the 
catalogue of "other" technologies. If BOFS is to be comprehensive, it cannot simply ignore the implications of this statement. Pragmatically, there is nothing a project like DIADEM can achieve where an application provider opts out of the standards and interoperability framework in order to deliver their own proprietary solutions. However, there is a need to consider whether these opt outs are forced because categories identified above fail to cover something users will perceive as significant form navigation or data entry behaviour.

When it was developed, the W3C standards body considered the form $<$ FORM $>$ and input control elements in HTML 4.0 [W3C 1999] combined with hyperlinks, to be adequate for the needs of online transactions and forms of all common types. However, W3C has reviewed the operation of the standard and the working draft Web Forms 2.0 [W3C 2006] defines the proposed extensions to the $<$ FORM $>$ element in HTML 5. Most of the recommended changes are richer behaviour or different renderings of the operations described above that don't change the user perception of form complexity.

Web Forms 2.0 does recommend the addition of a range control that can look like a slider or volume control and variation on a numeric type-in box by adding count-up and count-down buttons, an example of which can be seen in Figure 5.

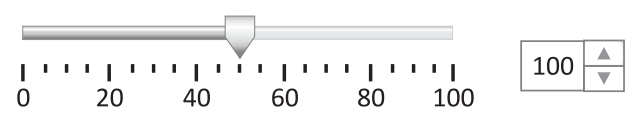

Figure 5. Slider control (left), count-up and count-down buttons (right).

In terms of evaluating and comparing existing forms, the five input behaviours and criteria above appear to be adequate for a wide range of services and applications. However, in the medium to long-term, additional criteria within BOFS8 will be needed to deal with slider and spinner controls.

\section{Utilising BOFS}

The BOFS criteria outlined in Section 4 were used during the course of the DIADEM project to assess online-forms in the UK, Italy, Norway. The goal of the exercise was to identify representative online-forms for use in the DIADEM user trials. Table 3 provides an example of eight online-form complexity records that were produced for a range of candidate UK online-forms. The selection of the eight online-forms that would subsequently be analysed using BOFS, was carried out by the DIADEM research team, who set out to identify candidate online-forms that represent a range of web-services from the public and private sectors, that older-adults may typically attempt to access within a home setting. Similar records were produced for Italian and Norwegian online-forms. The eight onlineforms selected for this study are presented in Table 3. Figure 6 provides an example of a First Great Western (FW) online-form for booking train tickets [2009].

\subsection{Online-form Selection Procedure}

A procedure, using the BOFS criteria, that was carried out for identifying the most appropriate online-forms for use within the DIADEM user trials is now presented. The procedure was carried out in three stages, and is exemplified by referring to the eight online-form complexity records presented in Table 3.

Stage one: Data collection. Data was collected for each of the BOFS criteria relating to a range of candidate online-forms. The collected data, representing the BOFS criteria for each candidate online-form, was then compiled into tabular format. This allowed researchers to carry out a comparison of the BOFS scores for each candidate online-form. Table 3 provides an example of a completed table for eight online-form complexity records produced for eight online-forms assessed in this study. In the case of the DIADEM project in the UK, the process of collecting values for each of the BOFS criteria was carried out by two researchers. As a precautionary measure, the two researchers met prior to carrying out the main task, and scored two online-forms together so that individual understanding of the scoring criteria may be discussed and aligned, to ensure that there was mutual agreement on the meaning of each of the BOFS criteria. Researchers reported that this was a straightforward process, and that the BOFS criteria (as described above) were clear 


\begin{tabular}{|c|c|c|c|c|c|c|c|c|}
\hline & EA & FBC & FW & $\mathrm{JC}$ & LBH & SB & SCC & TC \\
\hline BOFS1: No. of Pages in the form & 2 & 1 & 8 & 6 & 8 & 5 & 1 & 2 \\
\hline BOFS2: No. of pages requiring scrolling & 2 & 0 & 4 & 1 & 4 & 3 & 1 & 1 \\
\hline BOFS3: Provide current location & Yes & Yes & Yes & No & No & No & No & No \\
\hline BOFS4: Provide overall location & No & No & Yes & No & No & No & No & No \\
\hline BOFS5: No. of questions in the form & 26 & 14 & 20 & 9 & 60 & 23 & 38 & 19 \\
\hline BOFS6: Max No of possible responses & 58 & 14 & 24 & 27 & 60 & 23 & 38 & 3 \\
\hline $\begin{aligned} \text { BOFS7: } & \text { No. of responses requiring additional } \\
& \text { information }\end{aligned}$ & 0 & 1 & 3 & 0 & 5 & 3 & 1 & 0 \\
\hline BOFS8a: No. of drop-down boxes in the form & 4 & 1 & 14 & 5 & 14 & 11 & 4 & 2 \\
\hline No. of drop-down boxes requiring scrolling & 0 & 0 & 13 & 0 & 0 & 11 & 0 & 0 \\
\hline No. of options in dropdown-boxes & $\begin{array}{l}1 \times 7 \\
3 \times 10\end{array}$ & $1 \times 5$ & $\begin{array}{l}2 \times 6 \\
1 \times 7 \\
2 \times 4 \\
2 \times 11 \\
2 \times 12 \\
2 \times 31 \\
3 \times 3\end{array}$ & $\begin{array}{l}1 \times 3 \\
1 \times 4 \\
1 \times 5 \\
1 \times 8 \\
1 \times 29\end{array}$ & $\begin{array}{l}2 \times 31 \\
2 \times 67 \\
4 \times 59 \\
6 \times 12\end{array}$ & $11 \times 1$ & $\begin{array}{l}2 \times 2 \\
1 \times 3 \\
1 \times 4\end{array}$ & $\begin{array}{l}1 \times 4 \\
1 \times 8\end{array}$ \\
\hline BOFS8b: No. of radio buttons in the form & 0 & 7 & 3 & 0 & 12 & 3 & 8 & 0 \\
\hline No. of options in radio buttons & 0 & $\begin{array}{l}2 \times 2 \\
1 \times 3\end{array}$ & $\begin{array}{l}1 x 1 \\
1 \times 17 \\
1 \times 45 \\
\end{array}$ & 0 & $\begin{array}{l}9 \times 2 \\
1 \times 21 \\
2 \times 10 \\
\end{array}$ & $\begin{array}{l}2 \times 2 \\
1 \times 52\end{array}$ & $4 \times 2$ & \\
\hline BOFS8c: No. of check boxes in the form & 3 & 0 & 3 & 3 & 0 & 2 & 0 & 2 \\
\hline No. of options in check boxes & $\begin{array}{l}1 \times 1 \\
1 \times 3 \\
1 \times 40 \\
\end{array}$ & 0 & $\begin{array}{l}1 \times 1 \\
1 \times 2 \\
1 \times 5 \\
\end{array}$ & $\begin{array}{l}1 \times 1 \\
1 \times 5 \\
1 \times 6 \\
\end{array}$ & & $2 \times 1$ & 0 & $2 \times 1$ \\
\hline BOFS8d: No. of hyperlinks in the form & 0 & 0 & 7 & 1 & 0 & 2 & 0 & 4 \\
\hline No. of options in hyperlinks & 0 & 0 & $1 \times 18$ & $1 \times 2$ & 0 & $2 \times 1$ & 0 & $4 \times 1$ \\
\hline BOFS8e: No. of type-in boxes in the form & 15 & 1 & 4 & 4 & 42 & 15 & 27 & 9 \\
\hline $\begin{array}{l}\text { No. of type-in boxes requiring more than one } \\
\text { sentence }\end{array}$ & 1 & 0 & 0 & 0 & 4 & 0 & 0 & 0 \\
\hline $\begin{array}{l}\text { Key: } \\
\text { FW = First Great Western trains, ticket purchase (2009) } \\
\text { JC = Job Centre Plus application (2008) } \\
\text { SB = Sainsbury's home shopping registration (2009) } \\
\text { TC = Tesco's home shopping registration (2009) } \\
\text { LBH = London Borough of Hillingdon part time job vac } \\
\text { EA = EAGA warm front grant application (2009) } \\
\text { SCC = Sheffield City Council change of address form (2) } \\
\text { FBC = Fareham Borough Council disabled driver parkin } \\
\text { = Online-forms chosen based on respective criteri }\end{array}$ & $\begin{array}{l}\text { ncy ap } \\
\text { 009) } \\
\text { perm } \\
\text { values }\end{array}$ & $\begin{array}{l}\text { applica } \\
\text { (highes }\end{array}$ & $\begin{array}{l}\text { on form } \\
\text { or joint }\end{array}$ & $\begin{array}{l}\text { (2009) } \\
\text { ighest }\end{array}$ & lues) & & & \\
\hline
\end{tabular}

Table 3. Example complexity records for eight UK online-forms.

and applicable to all online-forms reviewed as part of this task.

Stage two: Comparing BOFS results. Members of the UK DIADEM research team, comprising of two HCI experts from Brunel University and two e-Inclusion consultants from Sheffield City Council, met to consider the values collected for each BOFS complexity records, and discussed the relative complexity of each of the respective online-forms. It should be noted that it is not the intention to total or combine the values generated for the BOFS complexity records, to produce cut-off scores that may be used to identify online-forms that are "complex" or "non-complex" per se, according to these totals. When we consider the almost unbounded number of online-forms that are presented on the web, it is unlikely that such cut-off values may be accurately identified, and indeed the long-term value of identifying cut-off values may also be questionable when considering the ever changing nature of online-form con- 
tent. The intended use of the BOFS complexity record is to serve as an aid in the decision making process, by providing a range of values that indicate how complex an online-form is according to the five ACE-R cognitive impairment categories. These scores may be taken into consideration when identifying an appropriate sample of online-forms for use within subsequent user trials, but may not be the only criteria taken into account. Indeed, which online-forms are most appropriate for a given set of user trials, will be defined by the research question that a given study is concerned with exploring. BOFS values are intended to provide insight into the complexity of online-forms according to the five ACE-R cognitive impairment categories. Ultimately, it is the researcher's decision, which online-forms are most appropriate for their study. For example, it may be the intention of the researcher to identify online-forms that are specifically complex according to one particular cognitive impairment category, such as attention and orientation (ACE-R1). In this scenario, it may be the case that online-forms that achieve a comparatively high score for the ACE-R1 category, and lower scores on the remaining ACE-R categories, could be considered as most appropriate for subsequent use within user trials. Alternatively, as was the case for the DIADEM project, researchers aimed to identify online-forms that were likely to be perceived as complex in all five ACE-R cognitive impairment categories. Hence, online-forms that scored highly in all of these categories were considered as more appropriate candidates for inclusion in subsequent user trials.

Ultimately, the researchers considered the requirements of the task at hand, and used the BOFS criteria values, to aid in the process of identifying appropriate online-forms. Specifically in the context of the DIADEM project in the UK, "round the table" discussions were held, comparing and contrasting the BOFS criteria values generated for eight online-forms. The actual online-forms used to populate the BOFS table were also made available during these discussions, and perused by the team of researchers as was seen necessary. Since each of the BOFS criteria represented a feature of the online-form that was likely to present a challenge to cognitively impaired older-adult users, the higher the score for each BOFS criteria, the higher the complexity of the online-form was considered to be for each respective criterion.

Stage three: Selecting appropriate onlineforms. The team of researchers made a final selection of candidate online-forms to be used in the user trials based on the BOFS criteria values and the needs of the project. As an example, in the context of the DIADEM project, the goal was to identify online-forms that presented the highest level of complexity for the users, so that a comprehensive range of DIADEM system requirements may be derived when users
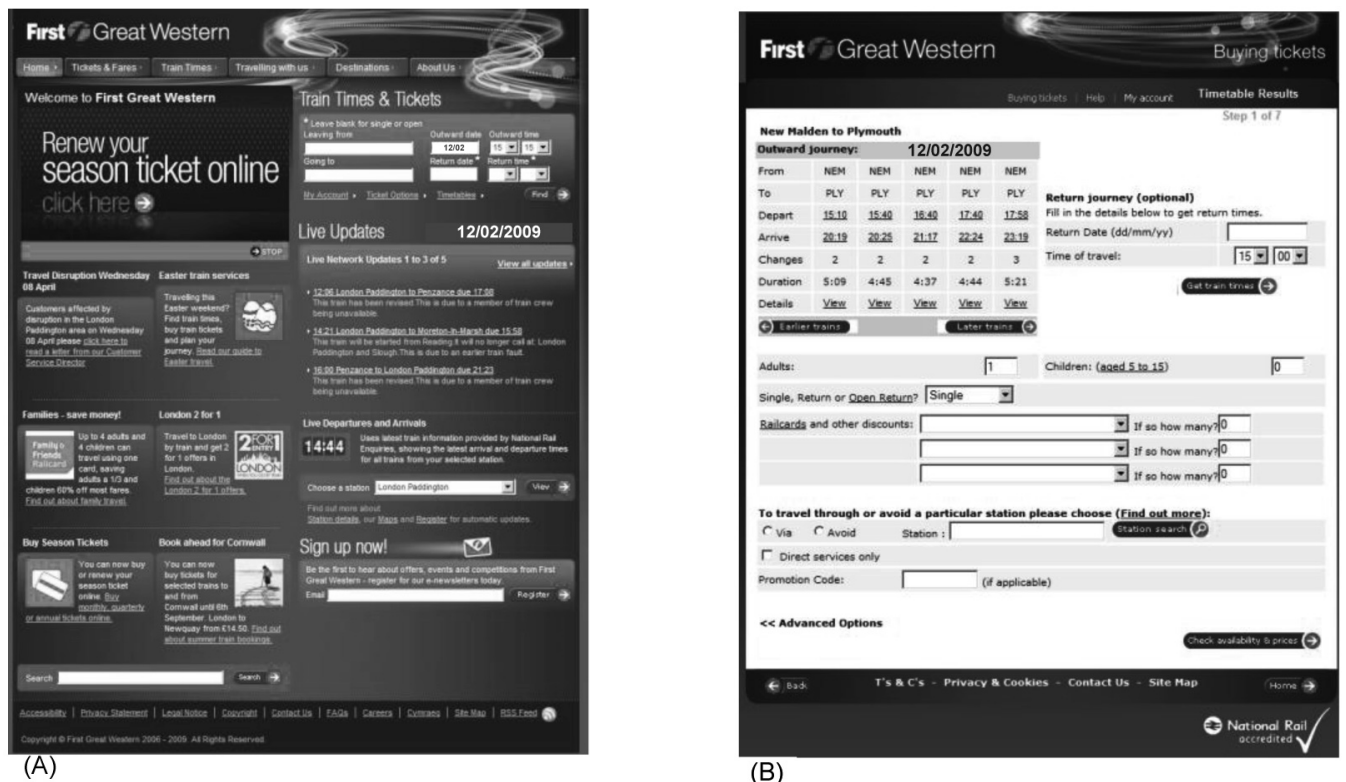

(B)

Figure 6. First Great Western ticket booking forms (A) opening page, (B) subsequent booking screen. 
interacted with the online-forms during user trials. The DIADEM team also had the provisor that online forms chosen for the user trials should contain a wide variety of input mechanisms (drop-down boxes, radio buttons, check boxes, hyperlinks and type-in boxes) as noted by BOFS $8 \mathrm{a}, 8 \mathrm{~b}, 8 \mathrm{c}, 8 \mathrm{~d}$, and $8 \mathrm{e}$.

Based on the eight example online-form complexity records presented in Table 3 , no individual online-form appeared to achieve the highest score for all BOFS criteria. Consequently, it was necessary to select a combination of the eight online-forms to ensure that the onlineforms used in the trials achieved comparatively high scores for all BOFS criteria categories. As a result it was apparent that selecting two onlineforms out of the eight would ensure that comparatively high values would be achieved for all BOFS criteria. These were the First Great Western trains, ticket purchase [2009] (FW) and London Borough of Hillingdon employment application [2008] (LBH). The FW onlineform achieved the highest or joint highest BOFS complexity scores in terms of BOFS1, BOFS2, BOFS8a, BOFS8c, and BOFS8d, in which it scored highest or joint highest for all of these criteria. The London Borough of Hillingdon employment application [2008] (LBH) onlineform did not provide detail relating to current location (BOFS3) or overall location (BOFS4) hence making the form filling process more cognitively challenging, and scored highest on the BOFS criteria that the FW online-form did not score highest on, these being BOFS5, BOFS6, BOFS8b, and BOFS8e. Therefore, based on the observation that the combination of these online-forms would result in users being exposed to online-form content that achieved the highest BOFS values within a trials setting, these two online-forms were deemed as being most appropriate for use within a DIADEM trials setting, given the need to expose users to complex online-forms for the purposes of the DIADEM project.

\section{Lessons Learned}

Developing BOFS was particularly challenging due to the lack of existing online-form complexity knowledge presented in existing literature specific to the cognitively impaired older adult user group. As a result, it was necessary to adopt a pragmatic and innovative methodological stance to overcome this challenge, and develop BOFS. In this section, we reflect on the effectiveness of BOFS in the light of the requirements of the DIADEM project and consider the methodological guidelines based on the experience of carrying out this research activity.

\subsection{Effectiveness of BOFS}

BOFS has successfully been used within the context of the DIADEM project. There have been a number of benefits associated with using such criteria within the DIADEM project. These are as follows:

- Having a set of criteria that can be used to tangibly represent the relative complexity of a range of online-forms as opposed to, for example, running a separate set of trials to establish complexity has been of great value to the project. Not least, it has meant that time and resource savings have been made, which have been put into good use elsewhere, such as running extensive user trials for functional and usability requirements capture.

- BOFS have provided a standardised measure of online-form complexity, which has proved to be applicable to a wide range of online-forms regardless of subject content or language. This has been a great benefit to the project, particularly when considering that the DIADEM project is running in three European countries. The use of BOFS has meant that all members of the team have been able to gain insight into the relative complexity of online-forms, regardless of the language the online-form was originally presented in. As a result, this has helped to ensure consistency across partner countries, in terms of the types and complexity of online-forms used within the respective trials.

- BOFS has provided tangible and comparable criteria of online-form complexity, which has meant that the risks of potential ambiguity and subjectivity when assessing onlineform complexity has been managed and considerably reduced. Having said this, subjective judgments have been taken, but these have been as a group, during the final selection stages of the assessment process, and therefore we feel the margin for error has 
been reduced. The clearly structured complexity criteria presented within BOFS, ensured that the group decision making process could be carried out with an added level of clarity and mutual understanding.

- BOFS is also useful for measuring onlineform complexity specific to the older adult user group. This is something that does not appear to have been done as yet in existing research, and thus has proved extremely valuable given the needs of the DIADEM project, as it would likely to be for new research into evaluating the usability of onlineform content for older adults.

- Since all criteria are recorded, BOFS has the added benefit of providing a dimension of traceability of selection results, that otherwise would not be available via other assessment methods. This may be of particular value in future research activity that builds on the activity carried out on the DIADEM project.

\subsection{Methodological Guidelines}

A number of lessons were learned as a result of designing and co-ordinating the collaborative consensus building activity carried out to develop the BOFS criteria. These may be considered as the following methodological guidelines:

- Recruitment of appropriately experienced and knowledgeable expert panel members is paramount to the feasibility of carrying out consensus building activity. Without a wide reaching network of partner organisations that possess a range of appropriate expertise, as was the case for the DIADEM project, embarking on such an activity should be carefully considered against the effort that would be required to recruit such members.

- It is important to recruit panel members that are motivated and committed to the consensus building task. Prior to meeting, panel members are expected to invest time and resources into orientating themselves with the task at hand, which includes reading and understanding a range of information related to the task. Without motivation and commitment to the task, it is unlikely that the time and resource overhead would be appropriately catered for by panel members. In the case of this study, a key success factor was that panel members were already DIADEM consortium members, and hence had a vested interest in the success of the DIADEM project.

- Maintaining clear and regular communication with panel members prior to the consensus building meeting is a critical success factor. In particular, members often required support and assistance in orientating themselves with the nature of the task, and ensuring that their understanding of the nature of the task was aligned with other panel members. Considerable effort should be invested in ensuring that panel members are appropriately supported prior to meeting and that all queries are answered and issues discussed, so as to ensure that maximum progress is made on the day of meeting.

- All members should be provided with appropriate background material prior to meeting. This ensures that all members are equally well informed, and able to effectively communicate with each other in an informed and mutually comprehensible fashion. In the case of the DIADEM experience, this was particularly important, since experts from a range of areas of expertise were members of the review panel.

- In terms of the number of members involved in the process, we found that a panel of 12 experts was sufficiently low in number to allow each individual participant to contribute individually to the proceedings. However, this did not occur naturally, it is important to have a designated facilitator, to ensure that the process is mediated and a sufficiently balanced discussion occurs, taking into account all experts opinions.

\section{Conclusions}

In this paper, a need has been identified, for the development of criteria that serve as a means of assessment of online-form complexity for the older adult user group. As a result, the BOFS criteria have been developed and presented. To demonstrate how the BOFS criteria can be used within a real world context, the procedure employed to assess online-form complexity on the DIADEM project was then presented. Finally, 
the benefits of utilising the BOFS criteria as an aid to identifying complex online-forms within the context of the DIADEM project have been discussed and methodological guidelines have been presented relating to the experiences of carrying out a consensus building task with expert panel members. BOFS has been particularly useful as an aid to evaluating the onlineform complexity specific to the older adult user group. This is something that does not appear to have been done as yet in the existing literature, and thus was extremely valuable in meeting the needs of the DIADEM project and achieving DIADEM project goals. To date, BOFS criteria values have been generated for a range of online-form content in three languages have which has also demonstrated that BOFS appears to be a versatile and applicable in a wide range of contexts.

\section{References}

[1] Administration, U. S. G. S. Section 508 Standards, (1998).

[2] M. S. Albert, C. R. SAVAge, D. Blazer, K. Jones, L. Berkman, T. SEeman And J. W. Rowe, Predictors of cognitive change in older persons: MacArthur studies of successful aging. Psychology and Aging 10, 4 (1995), 578-598.

[3] A. ARCH, Web Accessibility for Older Users: A Literature Review, (W3C2008).

[4] B. F. AtKinson, T, O. Bennet, G. S. BAHR AND M. W. NELSON, Development of a Multiple HeuristicEvaluating Table (MHET) to Support Software Development and Usability Analysis. Springer, Hiedelberg, (2007).

[5] M. BAYLES, Designing online banner advertisements: should we animate? ACM Press (2002), 363-366.

[6] N. M. M. Black, D. Lamping, M. Mckee, C. SANDERSON, J. ASKHAM AND T. MARTEAU, Consensus development methods: a review of best practice in creating clinical guidelines. Journal of Health Services Research and Policy 4, 4 (1999), 236-248.

[7] British-Airways British Airways travel pages, British Airways, (2008).

[8] T. Buxton, A. TARrell And A. Fruhling, Heuristic Evaluation of Mission-Critical Software Using a Large Team. Springer-Verlag, Berlin Heidelberg,(2009).

[9] A. Chevalier AND N. BonNARDEL, Articulation of web site design constraints: Effects of the task and designers' expertise. Computers in Human Behavior 23, 5 (2007), 2455-2472.
[10] H. Christensen, What cognitive changes can be expected with normal ageing? Australian and New Zealand Journal of Psychiatry 35 (2001), 768-775.

[11] T. CRIBBIN AND C. CHEN, Exploring cognitive issues in visual information retrieval. IOS Press (2001), 166-173.

[12] E. De Kock, J. VAN BILJON AND M. Pretorius, Usability evaluation methods: Mind the gaps, ACM Press, Johannesburg, South Africa (2009).

[13] Disability-Rights-Commission Inclusive design - A report by RICAbility (2005).

[14] S. M. Drucker, A. Glatzer, S. DE Mar, AND C. WONG, SmartSkip: consumer level browsing and skipping of digital video content. ACM Press (2002), 219-226.

[15] S. T. Dumais, E. CuTTRELl AND H. CHEN, Bringing order to the web: optimizing search by showing results in context. ACM Press (2002), 277-283.

[16] EAGA-Group Warm front application form, EAGA Group (2009).

[17] K. V. ECHT, Designing Web-Based Health Information for Older Adults: Visual Considerations and Design Directives, Lawrence Erlbaum Associates Inc, Hillsdale, NJ, USA, (2001).

[18] A. FAABORG AND D. SCHWARTZ, Using Distributed Heuristic Evaluation to Improve Usability of Open Source Software. ACM Press, Atlanta, Georgia, USA, (2010).

[19] Fareham-Borough-Council Application form for disabled driver's parking bay, Fareham, (2009).

[20] S. Fernando, A. G. Money, A. D. Elliman AND L. LINES, Developing Assistive Web-base Technologies for Adults with Age Related Cognitive Impairments, Transforming Government: People, Process and Policy. Transforming Government: People, Process and Policy 3, 2, (2009), 131-143.

[21] First-Great-Western Train ticket purchase, FirstGreat-Western (2009).

[22] M. F. Folstein, S. E. Folstein And P. R. MC Hugh, "Mini-mental state": A practical method for grading the cognitive state of patients for the clinician. Journal of Psychiatric Research 12, 3, (1975), 189-198.

[23] E. FrokJAer AND K. HornBAEK, Metaphors of Human Thinking for Usability Inspection and Design. ACM Transactions on Human-Computer Interaction 14, 4, (2008), 20-33.

[24] M. FuCHSBERGER, Ambient Assisted Living: Elderly People's Needs and How to Face Them. ACM Press, (31 October 2008), 21-24.

[25] D. Hawthorn, Possible implications of aging for interface designers. Interacting with Computers 12 , $5,(2000), 507-528$. 
[26] J. HeO, D. HAM, S. PARK, C. Song AND W. C. YoOn, A framework for evaluating the usability of mobile phones based on multi-level, hierarchical model of usability factors. Interacting with Computers 21, 4, (2009), 263-275.

[27] T. HollingSED AND D. G. NovicK, Usability inspection methods after 15 years of research and practice. ACM Press, (2007), 249-255.

[28] A. HolzINGER, Usability Engineering for Software Developers. Communications of the ACM 48, 1 (2005), 71-74.

[29] A. Holzinger, K. S. MuKasa AND A. K. NischelWITZER, Introduction to special thematic session: Human-Computer Interaction \& Usability for Elderly (HCI4AGING). Springer-Verlag, Berlin, Heidelberg, New York, (2008).

[30] A. Holzinger, G. Searle, T. Kleinberger, A. SEFFAH AND H. JAVAHERY, Investigating Usability Metrics for the Design and Development of Applications for the Elderly, Springer-Verlag, Berlin, Hiedelberg, New York, (2008).

[31] K. HornbaeK, Current practice in measuring usability: Challenges to usability studies and research. International Journal of Human-Computer Studies 64 (2006), 79-102.

[32] E. T. HVANnBerg, E. L. C. LAW AND M. K. LARUSDOTTIR, Heuristic evaluation: Comparing ways of finding and reporting usability problems. Interacting with Computers 19, (2007), 225-240.

[33] L. IsZO AND E. LANG, Heart period variability as mental effort monitor in human computer interaction. Behavious and Information Technology 19, 4, (2000), 297-306.

[34] Job-Centre-Plus Online job application form, JobCentre-Plus, (2008).

[35] T. Kleinberger, M. Becker, E. Ras, A. HOLZINGER, AND P. Muller, Ambient Intelligence in Assisted Living: Enable Elderly People to Handle Future Interfaces. Springer-Verlag, New York, (2007).

[36] S. KURNIAWAN AND P. ZAPHIRIS, Research-Derived Web Design Guidelines for Older People. ACM Press, (2005).

[37] A. J. LARNER, An audit of the Addenbrooke's Cognitive Examination (ACE) in clinical practice: 2 . International Journal of Geriatric Psychiatry 21, 7 , (2006), 698-699.

[38] L. LiNES , O. IKECHI , K. HONE AND T. ElLIMAN, Online form design for older adults: Introducing web-automated personalisation. HCI, (12 September 2006).

[39] L. Lines, O. IKeChI AND K. S. Hone, Accessing e-Government Services: Design Requirements for the Older User, Lecture Notes in Computer Science, vol. 4868. Springer Berlin / Heidelberg, (2007).

[40] L. LINES , Y. PATEL \& K. S. HONE, Online form design: Older adults' access to housing and welfare services. HCI, (7-11 September 2004), 21-22.
[41] C. Ling AND G. SAlvendy, Effect ofevaluators'cognitivestyleonheuristicevaluation: Field dependentandfieldindependentevaluators. International Journal of Human-Computer Studies 67, (2009), 382-393.

[42] London-Borough-Of-Hillingdon Online job application, (2008).

[43] J. McGrenere, R. M. BAeKer And K. S. BoOth, An evaluation of a multiple interface design solution for bloated software. ACM Press (2002), 163-170.

[44] S. MiLler AND C. JARRETT, Should I use a drop down? Four steps for choosing form elements on the Web, Effortmark Ltd, (2001).

[45] E. Mioshi, K. Dawson And J. Mitchell, The Addenbrooke's Cognitive Examination Revised (ACE$\mathrm{R})$ : a brief cognitive test battery for dementia screening. International Journal of Geriatric Psychiatry 21, 11, (2006), 1078-1085.

[46] A. G. Money, L. Lines, S. Fernando And A. D. ELLIMAN, e-Government Online-Forms: Design Guidelines for Older Adults in Europe. Universal Access in the Information Society 10, 1, (2011), p.p. Forthcoming.

[47] R. W. Morrell AND C. B. MAYHORN, A survey of world wide web in middle age and older adults. Human Factors 42, 2, (2000), 175-185.

[48] R. W. MORRELL, http://www.nihseniorhealth.gov: the process of construction and revision in the development of a model web site for use by older adults. Universal Access in the Information Society 4, 1, (2005), 24-38.

[49] R. W. Morrell, S. R. Dailey, M. Stoltz-Loike, C. FELdMAN, C. B. MAYHORN, K. V. ECHT AND K. I. PODANY, Older Adults and Information Technology: A Compendium of Scientific Research and Web Site Accessibility Guidelines, National Institute on Aging and National Library of Medicine, Bathesda, MD, USA, (2002).

[50] A. NASEEM, Dynamics of user needs analysis in redesigning as open learning website: A case from Pakistan. (2005), 503-507.

[51] J. NIELSEN, Heuristic evaluation, John Wiley \& Sons, New York, NY, USA, (1994).

[52] J. NIELSEN AND D. NORMAN, Web Usability for Senior Citizens: 46 Design Guidelines Based on Usability Studies with People Age 65 and Older. Nielsen Norman Group, (2002).

[53] A. Papazafeiropoulou, A. Pouloudi and A. POULYMENAKOU, Electronic commerce competitiveness in the public sector: the importance of stakeholder involvement. International Journal of Services Technology and Management 3, 1, (2002), 82-95. 
[54] R. C. Peterson, G. E. Smith, S. C. Waring, R. J. IVNiK, E. KoKMEN AND E. G. TANGelos, Aging, memory, and mild cognitive impairment. Intenational Psychogeriatrics 9, 1 (1997), 65-69.

[55] B. Reisberg, S. H. Ferris, M. J. De LeON, AND T. CROOK, The Global Deterioration Scale for Assessment of Primary Degenerative Dementia. American Journal of Psychiatry 139 (1982), 1136-1139.

[56] Sainsbury's Online shopping registration form, Sainsburys, (2009).

[57] S. SAYAgo AND J. BLAT, Some Aspects of Designing Accessible Online Forms for the Young Elderly. INSTICC Press, Barcelona, Spain, (2007).

[58] B. SCHNEIDERMAN, Designing the user interface. Addison-Wesley, Reading, MA, USA, (1998).

[59] A. SefFah, M. Donyaee, R. B. Kline, and H. K. PADDA, Usability measurement and metrics: A consolidated model. Software Quality Journal 14, (2006), 159-178.

[60] Sheffield-City-Council, Tell us you've moved, Sheffield, (2009).

[61] Z. TANG, T. JOHNSON, R. TINDALL AND J. ZHANG, Applying heuristic evaluation to improve the usability of a telemedicine system. Telemedicine Journal and E-Health 12, 1, (2006), 24-34.

[62] D. TE'EnI, J. CAREY AND P. ZHANG, HumanComputer Interaction: Developing Effective Organizational Information Systems. Wiley \& Sons, Inc, New York, USA, (2007).

[63] Tesco Online shopping registration form, Tesco, (2009).

[64] W3C HTML 4.0 Specification: Chapter 17, Forms Recommendation 24 December 1999.

[65] W3C Web Forms 2.0: Working Draft, (2006).

[66] W3C Web Content Accessibility Guidelines (WCAG) 2.0, (2008).

[67] D. WelchSLER, Manual for the Wechsler Adult Intelligence Scale: revised, Psychological Corporation, New York, NY, USA, (1982).

Received: August, 2009 Revised: May, 2010 Accepted: July, 2010

Contact address:

Dr Arthur G. Money

Department of Information Systems and Computing

Brunel University Uxbridge, UB8 3PH

$\mathrm{UK}$
DR ARTHUR G. MONEY received his MSc in Distributed Information Systems from Brunel University in 2000 before working as an e-Business Technology Consultant for Oracle Corporation. He returned to academia in 2004 and received his $\mathrm{PhD}$ in Human Computer Interaction from Brunel University in 2007. Since then, he has worked as a Research Fellow within the Department of Information Systems and Computing at Brunel University. Arthur is a professional member of the British Computer Society and his research interests include multimedia computing, human factors and user-centred design methods for the development and evaluation of interactive systems for improving quality of life.

DR SENAKA FERNANDO joined the School of Information Systems, Computing and Mathematics at Brunel University in the UK as a Research Fellow in 2001. He holds a PhD in Information Systems (Brunel University) and is a professional member of the British Computer Society. He has research interests related to organisational aspects of information systems, technology enabled service transformations in governments, health information systems, and diffusion of innovations. Senaka has published his work in a number of leading journals and conferences. Prior to returning to the academia and education, he worked in engineering and healthcare organisations for several years.

DR LORNA LINES joined the Department of Information Systems and Computing at Brunel University in 2000 as a full time researcher and part time $\mathrm{PhD}$ student, contributing to the multimodal-user interface design for the Millennium Home Project, which is led by Professor Heinz Wolff. In 2003, Lorna was awarded her PhD and appointed as a Lecturer in Information Systems and Computing at Brunel University. She has collaborated on a range of international research projects, and has recently been a co-investigator on the EU (FP6) DIADEM project, which aims to enable older adults to access government and commercial services, while supporting independent living. More generally, Lorna's research interests focus on the design and development of innovative, multimodal system interfaces for older adults to support and maintain independent living.

DR TONY ELLIMAN gained his Doctorate for research in medical information systems in 1989 and is now a Reader in Information Systems Architecture at Brunel University. His research interests are in the architecture and evaluation of information systems within the public sector and in particular the development of effective systems to support knowledge work. He co-ordinated the EU funded international project DIADEM and was a co-investigator in the Virtual Institute for Electronic Government Research (VIEGO). He is a regular conference chair and serves on the editorial boards of both the Journal of Enterprise Information Systems and Transforming Government: People, Process and Policy. Dr Elliman gained his original degree in electronics and electrical engineering while working with ICL before becoming a computer science lecturer in 1972. As a registered Chartered Engineer and Chartered IT Professional he has provided software development and consultancy services to government, the health sector, academic and private sector organizations, including DERA and the EU Commission. 